CEA and CK20 are two epithelial cell markers whose expression is retained in CRC. Detection of these markers in locations outside epithelial compartments-for example in the lymph nodes-indicates that disseminated tumor cells are present. The investigators devised real-time, quantitative, reverse transcriptase polymerase chain reaction (qRTPCR) assays for CEA and CK20 mRNAs. They then assessed the value of the assays in the detection of disseminated tumor cells in lymph node samples from 51 CRC patients.

Compared with noncancer controls, levels of CEA and CK20 mRNA were significantly elevated in the CRC samples, with those from Dukes' Stage $C$ and $D$ patients showing the highest values. The CEA assay was superior in terms of specificity, although the CK20 assay may prove useful in detecting cells with low CEA expression.

Once clinically relevant cut-off levels are established, it is hoped that these methods will contribute to improved outcomes in CRC treatment, by helping to identify patients for whom adjuvant chemotherapy is appropriate.

Original article Öberg ÅNV et al. (2004) Detection of occult tumour cells in lymph nodes of colorectal cancer patients using real-time quantitative RT-PCR for CEA and CK20 mRNAs. Int J Cancer 111: 101-110

\section{Hepatocellular carcinoma in the elderly}

The incidence of hepatocellular carcinoma (HCC) is on the rise in a number of countries, partly because of an increase in the rate of hepatitis infection. As the population ages, the proportion of elderly HCC patients is likely to grow. Dohmen and colleagues have carried out a retrospective study to compare the clinical and prognostic characteristics of elderly HCC patients with those of their younger counterparts.

HCC patients consecutively diagnosed during a 12-year period were divided into two groups: the 'elderly' group $(n=36)$ aged 80 years or older and the 'non-elderly' group $(n=668)$. Clinical variables (sex, hepatitis C and hepatitis B markers, concentration of serum alpha-fetoprotein, diameter and number of tumors, Child's grade, presence of portal thrombosis, histological grade, types of treatment and survival) were compared between the two groups and prognostic factors were identified.
All clinical features-includi ng survival rate-were similar between the two groups. The elderly group revealed presence of portal thrombosis as the only significant prognostic factor, whereas prognosis in the non-elderly group was influenced by nonsolitary tumors, Child's grade of B or C, tumor diameter of $>3 \mathrm{~cm}$ and presence of portal thrombosis.

Since age did not appear to influence survival rate in this study, the authors propose that patients with HCC should be selected for aggressive treatment strategies based on their risk factors, irrespective of age.

Original article Dohmen K et al. (2004) Optimal treatment strategy for elderly patients with hepatocellular carcinoma. J Gastroenterol Hepatol 19: 859-865

\section{New device aids colon surgery}

Obstructing left-sided colon cancer can be treated surgically using a one-stage procedure, involving resection of the tumor and primary anastomosis with on-table antegrade irrigation. Conventional irrigation is problematic, however, and does not allow on-table colonoscopy. Park et al. have recently reported on their multicenter, prospective study of a new device, which has been designed to overcome these problems.

The investigators studied 151 patients with obstructing left-sided colon cancer. All patients underwent resection, primary anastomosis and on-table irrigation using the new intraoperative colonic irrigator ( $\mathrm{NICl}$ ) from the Korean company MITech. Half of the patients also underwent on-table colonoscopy by way of the device.

The time taken for irrigation was relatively short compared with conventional means, and a larger volume of fluid was delivered. There was a relatively low rate $(1.3 \%)$ of anastomotic leakages, suggesting that the procedure is safe. On-table colonoscopy allowed the detection of synchronous polyps in 35 (47\%) patients and led to extended resection due to malignancy of necrosis in $13(17 \%)$ cases.

The authors conclude that the $\mathrm{NICl}$ device enables quick and easy on-table irrigation and colonoscopy, which should facilitate the one-stage resection and primary anastomosis procedure.

Original article Park UC et al. (2004) Single-stage procedure with intraoperative colonoscopy and colonic irrigation in patients with obstructing left-sided colonic cancer. Int J Colorectal Dis 19: 487-492 